\title{
Domperidone-Related Acute Dystonia in a Young Child With Underlying Abnormal Electroencephalogram
}

\author{
Kai Lun Tang, c , Fauzana A. Mokhti ${ }^{\mathrm{b}}$, Jing Ying Fong ${ }^{\mathrm{a}}$, \\ Hunainah Khairul Anwar ${ }^{\mathrm{a}}$
}

\begin{abstract}
Domperidone is the preferred treatment for vomiting and gastroesophageal reflux in pediatric population. It is known to have lesser side effects compared to metoclopramide and is rarely associated with extrapyramidal side effects. We report a case of acute dystonia in a 4-year-old girl that occurred after given two doses of domperidone. She initially presented to the emergency department for persistent vomiting and treated with syrup domperidone, ranitidine and oral rehydration salts. Approximately $24 \mathrm{~h}$ after last dose of oral domperidone, she developed five episodes of abnormal movement, i.e. stiffness over bilateral upper and lower limbs with eye staring to one direction without blinking. Each episode lasted for less than 10 $\mathrm{s}$ and patient did not lose consciousness. No history of head trauma or any family history of neurological diseases was documented. Patient was admitted for workup to rule out seizure. In ward, she developed another two brief episodes of similar presentations which resolved spontaneously without any treatment. No re-occurrence of similar episodes throughout the 3 days of hospitalization was noted. Computed tomography scan of brain confirmed no acute intracranial bleed or focal brain lesion. Electroencephalogram done during subsequent clinic follow-up noted some abnormal records due to increase in slow delta activity, but parents did not consent for magnetic resonance imaging. Patient remained well on biannual follow-up without any seizure or dystonia episode. Rare adverse event involving the central nervous system should be carefully evaluated although product label for domperidone did not mention precaution of use in patients with underlying cerebral abnormalities or epilepsy.
\end{abstract}

Keywords: Domperidone; Acute dystonia; Adverse drug reaction; Abnormal electroencephalogram

Manuscript submitted March 10, 2020, accepted March 18, 2020

Published online June 18, 2020

aPharmacy Department, Seberang Jaya Hospital (Penang), Ministry of Health, Malaysia

bPediatric Department, Seberang Jaya Hospital (Penang), Ministry of Health, Malaysia

${ }^{\mathrm{c} C}$ Corresponding Author: Kai Lun Tang, Pharmacy Department, Seberang Jaya Hospital (Penang), Ministry of Health, Malaysia.

Email: tangkailun@hotmail.com

doi: https://doi.org/10.14740/ijcp364

\section{Introduction}

Domperidone is an antiemetic drug licensed for use in treatment of nausea, vomiting, dyspepsia and gastroesophageal reflux among pediatric population. Compared to metoclopramide, domperidone has a better safety profile and is rarely associated with extrapyramidal side effects because it does not effectively cross the blood-brain barrier under normal circumstances. Paediatric Protocol for Malaysian Hospital (4th Ed, 2019) did not specifically support the use of a particular prokinetic in treatment of gastroesophageal reflux disease, but majority of pediatricians favored domperidone over metoclopramide. Movement disorders, e.g. acute dystonia, parkinsonism, akathisia and tardive dyskinesis, can be caused by antiemetic medications although these side effects are very rarely reported [1-3]. Clinical manifestation is involuntary contraction of various muscles, affecting different areas, i.e. the oromandibular area, limbs, eyes, etc., which results in abnormal movements $[4,5]$. Dystonia of the oromandibular area may cause involuntary spasm of the jaw, lips and tongue muscles, affecting patient's swallowing, chewing capability or speech [5].

The 4-year-old girl we had with us developed acute dystonia which involved the eyes and limbs. Her parents described it as brief episodes of abnormal stiffness of bilateral upper and lower limbs with eyes gazing into one direction. It was never thought to be a side effect of domperidone. Despite being given in the recommended doses and interval, this very rare neurological complication of domperidone occurred. Further investigation found no drug-drug interaction between co-administration of domperidone with ranitidine and paracetamol. No traditional or complimentary medicine was involved in this case.

\section{Case Report}

A 4-year-old girl, weighing $17 \mathrm{~kg}$, presented to the emergency department (ED) with history of on and off fever and persistent vomiting for the past 3 days. She was later discharged home with syrup paracetamol, syrup domperidone, oral ranitidine and oral rehydration salts for treatment of acute viral gastroenteritis. Vomiting subsided after two doses of domperidone $(0.3 \mathrm{mg} / \mathrm{kg} /$ dose); hence the mother discontinued domperidone on the following day. Approximately $24 \mathrm{~h}$ after the last dose of oral dom- 
peridone, the girl developed five episodes of abnormal movements, i.e. stiffness over bilateral upper limbs and lower limbs with eyes staring into one direction without blinking and not responding to call. Each episode lasted for less than $10 \mathrm{~s}$. Parents claimed no drooling of saliva, no perioral cyanosis, no urinary or bowel incontinence, no change in behavior, abnormal speech or any obvious hallucination. The girl was brought to hospital and immediately admitted for further investigations to rule out seizure. There was no significant history of head trauma, febrile seizure or family history of any neurological diseases. Patient has no known medical illnesses and no known drug or food allergy. All immunization is completed up to age. Upon admission to ward, patient developed another two brief episodes $(<5$ s) of stiffness of the right hand with uprolling eye balls which resolved spontaneously without any treatment.

On admission, patient was alert, conscious, orientated but appeared lethargic. Vital signs were all normal (blood pressure (BP) 109/79 mm Hg, $\mathrm{SPO}_{2}$ 98\%, pulse rate regular 106 beats/min, respiratory rate 30 breaths/min, temperature 37.3 $\left.{ }^{\circ} \mathrm{C}\right)$. Physical examinations noted tongue was moist, bilateral tonsils grade II mildly injected without exudates, and no lymphadenopathy. Review of systems did not find any abnormality except that muscle tone on left and right upper limbs and lower limbs were normal with slight reduced in power $(3 / 5)$. Venous blood gas was taken to rule out acidosis caused by prolonged vomiting and all parameters were within normal limit ( $\mathrm{pH} 7.433, \mathrm{PCO}_{2} 28.8 \mathrm{~mm} \mathrm{Hg}, \mathrm{PO}_{2} 50.5 \mathrm{~mm} \mathrm{Hg}$, cHCO- $21 \mathrm{mmol} / \mathrm{L}$, base excess (BE) $-4.6 \mathrm{mmol}$ ). Hypoglycemia and hypocalcemia fit were ruled out with a normal reflow of $3.9 \mathrm{mmol} / \mathrm{L}$ and normal calcium level of $2.26 \mathrm{mmol} / \mathrm{L}$. Other parameters, i.e. full blood count (hemoglobin $(\mathrm{Hb}) 12.9 \mathrm{~g} /$ dL, white blood cell count (WBC) $7.3 \times 10^{9} / \mathrm{L}$, platelet 286 $\times 10^{9} / \mathrm{L}$ ), renal profile (urea $5.1 \mathrm{mmol} / \mathrm{L}$, sodium $\left(\mathrm{Na}^{+}\right) 136$ $\mathrm{mmol} / \mathrm{L}$, potassium $\left(\mathrm{K}^{+}\right) 4 \mathrm{mmol} / \mathrm{L}$, serum creatinine $(\mathrm{SrCr}) 39$ $\mu \mathrm{mol} / \mathrm{L})$, magnesium $\left(\mathrm{Mg}^{2+}, 0.81 \mathrm{mmol} / \mathrm{L}\right)$, phosphate $\left(\mathrm{PO}_{4}^{-}\right.$, $1.26 \mathrm{mmol} / \mathrm{L})$, albumin $(39 \mathrm{~g} / \mathrm{L}$ ) and $\mathrm{C}$-reactive protein (CRP, $1.4 \mathrm{mg} / \mathrm{L}$ ) were all within normal limit. Dengue NS1 antigen, immunoglobulin $\mathrm{M}(\mathrm{IgM})$ and immunoglobulin $\mathrm{G}(\mathrm{IgG})$ tested all negative. Developmental progress was up to age (can go up staircases with one step each foot, able to draw circle, able to feed herself and talk three words sentences). An urgent computed tomography (CT) scan of brain was done to rule out intracranial pathology and the findings confirmed no acute intracranial bleed, no focal brain lesion and no acute hydrocephalus. This further strengthened the possibility of domperidoneinduced acute dystonia.

Patient was afebrile in ward and did not have recurrent episodes of acute dystonia throughout the 3 days of hospitalization after discontinuation of the suspected offending medication domperidone. Electroencephalogram (EEG) was done after 3 months and findings noted some abnormal records due to increase in slow delta activity, admixed with occasional medium-high amplitude sharp waves variably over the right posterior quadrants. However, parents did not consent for magnetic resonance imaging (MRI) despite advice from pediatric neurologist. Patient remained well at 3-month and 12-month follow-ups without developmental delay or any documented episodes of seizure or dystonia.

\section{Discussion}

Domperidone is commonly prescribed for children with gastroesophageal reflux disease. Based on our hospital adverse drug reactions (ADRs) database since the past 5 years, extrapyramidal side effects such as dystonias, akathisia and tardive dyskinaesia have never been reported among infants and very young children. On literature search, only very few case reports of domperidone-induced extrapyramidal side effects were reported $[3,6]$. Although Derinoz and Caglar reported that the most frequent causes of drug-induced dystonic reactions in patients presented to the ED were antiemetics $(\mathrm{n}=24$, $43.6 \%$ ), all the cases involved metoclopramide [7].

Diagnosing domperidone-induced dystonia in pediatric population is challenging because this side effects is very rare. Dystonias in children can be associated with multiple factors, which include ischemic brain injury during or around the perinatal period, central nervous infections, reactions to certain drugs, brain trauma, or various vascular abnormalities. It can result from other illnesses affecting the central nervous system (CNS). Moreover, acute dystonic reactions may be confused with partial seizure and central nervous infection in young children. Diagnosis of drug-induced dystonia is made usually based on clinical judgement supported by detail history of intake of drug with tendency of causing extrapyramidal side effects. The ultimate diagnosis of drug-induced dystonia may be preceded by unnecessary use of antiseizure medications, a series of invasive investigations, i.e. multiple blood takings, lumbar puncture for cerebrospinal fluid (CSF) analysis, and imaging procedures (radiographic, or magnetic resonance). Hence, it is crucial for clinicians to be mindful of this very rare side effect of domperidone in order to diagnose drug-induced acute dystonia at earlier stage.

In our case, an urgent CT scan of brain was done to rule out intracranial pathology and the findings confirmed no acute intracranial bleed, no focal brain lesion and no acute hydrocephalus. Parents did not consent for lumbar puncture for CSF analysis and microbiology test. After a series of blood investigation, this adverse reaction was discussed with house pharmacist to screen for possible drug-related adverse effect presenting as dystonia. Based on Naranjo scale [8], the relationship score was "probable" after other possible causes of dystonia were ruled out. This probable adverse reaction relationship with domperidone was further strengthened with scoring using WHO Uppsala Monitoring Centre criteria.

Domperidone for this child was prescribed at lower therapeutic dose of $0.3 \mathrm{mg} / \mathrm{kg} / \mathrm{dose}$ and no overdose of domperidone was suspected. From previous case reports, onset of acute dystonia was not immediate, but occurred after intake of several doses, which was suggestive of a delayed presentation [3, $7,9]$. In this case, the onset of dystonic reactions was $24 \mathrm{~h}$ after the ingestion of second dose. From the pharmacokinetic perspective, acute dystonia did not occur when domperidone concentration was at its peak. Half-life of domperidone in healthy patient is estimated to be between 7 and $9 \mathrm{~h}$ [10]. In our case, this means that when acute dystonia occurred, the serum concentration of domperidone could have been at its trough concentration. The reactions resolved after seven brief episodes of acute dystonic reactions within several hours. No 
active intervention was done in view that patient did not lose consciousness and each episode lasted less than $10 \mathrm{~s}$. Furthermore, it was reported that drug-induced acute dystonias are usually reversible [11] without serious neurological sequelae.

We did not attempt the rechallenge test due to the troublesome nature of this reaction. Patient remained well on biannual follow-up. However, the abnormal findings on EEG suggestive of an increase in slow delta activity, admixed with occasional medium-high amplitude sharp waves variably over a specific region on the brain changed our thought towards patient's underlying condition. Hence, the patient was scheduled for further investigation using MRI but parents did not consent for the neuroimaging procedure. Parents were advised regarding the risk of epilepsy and were informed about the plan to start antiepileptic if the child presented with seizure. It was well aware to all that metoclopramide is contraindicated in patients with epilepsy in view that the frequency and severity of seizures may be increased. Still, domperidone-related CNS adverse effects remained rare and not well known of. Although there is no product label mentioning practice of precaution when using domperidone in patients with underlying cerebral abnormalities or epilepsy, cases with rare adverse event involving the CNS should be carefully evaluated. Lowest effective dose of the drug should always be considered when used among pediatric population. Awareness of the potential for certain drugs to cause dystonic reactions is important in making diagnosis of this movement disorder.

\section{Conclusion}

Drug-induced acute dystonia can be a differential diagnosis in infants and young children with movement disorder or seizure-like activity. Detail history about patient medication use and withdrawal of suspected causative medication should be considered before instituting interventions and more invasive investigation. EEG test may be helpful in capturing any abnormal brain electrical activity and revealing other underlying problems which could have been related to acute dystonia. Early diagnosis in these cases reduces the time and cost of management while improving the prognosis.

\section{Acknowledgments}

We would like to thank the Director General of Health Malaysia for his permission to publish this article.

\section{Financial Disclosure}

The authors have indicated they have no financial relationships relevant to this article to disclose.

\section{Conflict of Interest}

All authors have no conflict of interest to disclose.

\section{Informed Consent}

Written consent was obtained from patient's care-giver (mother).

\section{Author Contributions}

Tang Kai Lun and Dr. Fauzana A. Mokhti developed the conceptual framework for case-based report, supervised data collection, drafted the initial manuscript, review and revised the manuscript. Fong Jing Ying and Hunainah Khairul Anwar registered the case report on National Medical Research Registry (NMRR), did extensive literature review, in charge of acquisition of clinical and laboratory data, analyzed and interpreted all the relevant clinical and laboratory data and drafted the discussion. All authors approved the final manuscript as submitted and agree to be accountable for all aspects of the work.

\section{Data Availability}

The authors declare that data supporting the findings of this study are available within the article.

\section{Abbreviations}

CT: computed tomography; EEG: electroencephalogram; MRI: magnetic resonance imaging; CNS: central nervous system; ED: emergency department; BP: blood pressure; $\mathrm{SPO}_{2}$ : pulse oximetry; SrCr: serum creatinine; $\mathrm{Hb}$ : hemoglobin; WBC: white blood cell count; WHO: World Health Organization; CRP: C-reactive protein; CSF: cerebrospinal fluid; IgM: immunoglobulin M; IgG: immunoglobulin G; ADR: adverse drug reaction

\section{References}

1. Casey DE. Neuroleptic drug-induced extrapyramidal syndromes and tardive dyskinesia. Schizophr Res. 1991;4(2):109-120.

2. Pellegrino M, Sacco M, Lotti F. [Extrapyramidal syndrome caused by moderate overdosage of domperidone. Description of a case]. Pediatr Med Chir. 1990;12(2):205206.

3. Dhakal OP, Dhakal M, Bhandari D. Domperidone-induced dystonia: a rare and troublesome complication. BMJ Case Rep. 2014;2014.

4. Dystonias Fact Sheet. NINDS-National institute of health. http://www.nids.nih.gov. (Accessed date: 25 March 2019).

5. Dystonia: causes,types symptoms and treatment. http:// www.webmd.com. (Accessed on: 25 March 2019).

6. Franckx J, Noel P. Acute extrapyramidal dysfunction after domperidone administration. Report of a case. Helv 
Paediatr Acta. 1984;39(3):285-288.

7. Derinoz O, Caglar AA. Drug-induced movement disorders in children at paediatric emergency department: 'dystonia'. Emerg Med J. 2013;30(2):130-133.

8. Naranjo CA, Busto U, Sellers EM, Sandor P, Ruiz I, Roberts EA, Janecek E, et al. A method for estimating the probability of adverse drug reactions. Clin Pharmacol Ther. 1981;30(2):239-245.
9. Shahbaz A, Elahi K, Affan M, Shahid MF, Sabir A. Acute dystonia secondary to domperidone in a pediatric patient. Cureus. 2018;10(11):e3587.

10. Product Information: Motilium(R), domperidone maleate. Janssen-Ortho, Toronto, Ontario, Canada (PI revised 1999) reviewed $7 / 2000$.

11. Medication induced dystonic reactions. https://emedicine.medscape.com/article/814632-overview. 